\title{
IGDA Finland Hubs and their role in local game development
}

\author{
Lauri Komulainen \& Olli Sotamaa
}

Tampere University

\section{FULL REFERENCE}

Lauri Komulainen and Olli Sotamaa. 2020. IGDA finland hubs and their role in local game development. In Proceedings of the 23rd International Conference on Academic Mindtrek (AcademicMindtrek '20). Association for Computing Machinery, New York, NY, USA, 92-99. DOI:https://doi.org/10.1145/3377290.3377294

\begin{abstract}
Game development is often considered to be a community-based industry, where social networks and flow of information are vital to both individual and company development. The International Game Developers Association (IGDA) works to promote and support individual game developers by hosting local monthly gatherings worldwide. This study investigates the effects of the IGDA Finland hubs in Tampere and Kajaani. By interviewing the attendees to the IGDA events in the respective areas we aim to understand how an IGDA hub affects the local game development scene, and how the attendees perceive the uses of the events.
\end{abstract}

The results show that IGDA Finland events can attract a diverse group of people with variety of motivations. Aggregating different insights and accelerating the flow of information seems important for people in very different positions. At the same time, the felt benefits for individuals seem to vary significantly based on the career phase and the expert role. Overall, IGDA gatherings are importantly connected to modes of learning in communities of practice, as they can initiate different communal processes that then continue outside the immediate meetings.

\section{Introduction}

With the advent of digital distribution platforms, accessible development tools and new emerging audiences, game development now happens in most parts of the world. It appears that the current videogame ecosystem is at the same time extensively global and intensely localised [1]. While the general trends around game making appear similar in different parts of the world, the spatial distribution of the games industry is importantly connected to the local histories and networks, as well as financial, cultural and labour markets [2].

Until recently most academic studies of the games industry have focused on key hubs of game development in North America and Asia [2]. Therefore, it has been inspiring to observe how in the past few years carefully crafted situated studies of regional game development environments have started to appear. In addition to leading manufacturers, dominant publishers, and large-scale domestic markets, scholars have set sights on exploring national game development histories [3,4,5], local development cultures [6,7] and particular game studios $[8,9]$.

This article draws inspiration from this recent emergence of game production studies, putting special focus on the informal networks between individual developers. Our study looks at the local hubs of International Game Developer Association. We specifically investigate two hubs located in Finland, namely Kajaani and Tampere. 
We want to understand, what are the different roles the IGDA events play in the local game development environments. The focus is on a) attendee demographics and motivations, b) common activities within the meetings, and c) the forms of learning associated with hub activities.

Finland is an apt object of study. Low hierarchies and open sharing of information are often associated with the Finnish game industry and the relaxed networking events like IGDA Gatherings are often mentioned as key hubs for bringing people regularly together $[10,11]$. Given this context, we are interested in learning how IGDA Finland activities affect the local game development scenes and especially the social and communal aspects associated with them.

While IGDA reports and white papers are often cited in academic studies, IGDA itself has been scarcely studied. As far as we know, no published studies have investigated the IGDA chapters and hubs and their relevance to the local game development scene. In this respect, the study at hand can provide entirely new insights to the communal structures and networks of game development. One of the authors is a co-founder and previous leader of the IGDA Finland Kajaani hub and an active member of the IGDA Tampere hub. The other author has visited IGDA meetings in several different towns and e.g. organized seminars in collaboration with IGDA Finland. In this respect, we have first-hand experience of the central IGDA hub activities like hosting gatherings, inviting guest speakers and seeking sponsors. We believe this background has prepared us well to ask relevant questions and to put the collected data into right contexts.

\section{Theoretical background}

In most cases, professional game development is a group effort. For any larger game project to succeed, one needs to find a group of somewhat like-minded people. Variety of different skillsets and specializations required in game development creates an additional challenge. In most cases, some sort of professional or semi-professional networks are needed to identify the potential team members. It is not uncommon that personal networks between professionals within the same field or simply by people with similar interests are extended to organizational level [21].

Prior research in the area indicates that the existing industries and other contextual factors affect the evolution of the game industry in particular areas. Japanese video-game industry has drawn from e.g. toys, consumer electronics, comics and animated-films, whereas the US industry evolved mostly from arcades and personal computers. At the same time, the UK game industry developed mostly from autodidact programmers and "bedroom coders" [22]. In the Nordic countries, early game developers did not get much support from existing electronics companies or other cultural industries. Instead, most upcoming game developers trained their skills within the hobbyist circles, including the demoscene [15]. More recently, the existing information technology industries have supported the growth of the game industry by providing e.g. seasoned programmers to the new industry [23].

Also the importance of so called "industry cocktail parties" where social networks are created and maintained has been pointed out [8]. Individuals who attend these gatherings are often more likely to find themselves a team, employment or even a publishing deal than those who don't. These kinds of events enable idea-sharing and inspiration via social exchange between the so called "upper ground" - existing game development companies and the "underground" aka indie developers outside said large companies [24]. In this respect, communities like IGDA can be seen as a creative middleground between the two worlds of game development. 
In this article, we will analyse the local IGDA as a community of practice (CoP). This concept, referring to a group of people who share a craft or a profession, was first proposed by Lave and Wenger [25] and later significantly expanded by Wenger [26]. Communities of practice provide a platform for forming identities, learning and finding meaning. CoPs can exist in physical settings, for example, a lunchroom at work, a field setting, a factory floor, or elsewhere in the environment, but members of CoPs do not have to be co-located. Members can also form a "virtual community of practice" [27] when they mostly collaborate online.

The main idea is that professional communities emerge around common practices [26]. Learning within the community of practice is explained as something that happens during the time that one spends inside said community. Such as learning important aspects of the culture of the community, the tools of the trade and the means of using them are learned when working in the community of practice. Later studies have shown how a 'community of practice' often involves knowledge management and creation in a mutual area of practice: creating and transferring knowledge is often best achieved in social spaces and in face-to-face interactions [28].

Game development as an activity consists of a set of practices that are commonly learned both at school, at home and at the workplace. Game developer is never complete, but skills are constantly updated and improved.

Previously, finding people with similar interest has been elemental for one's career [15]. Today online resources provide important opportunities for learning, but the role of local community should not be undermined [6]. In this sense, the idea of community of practice is a useful lens to observe the local activities. While some of the connections may seem tenuous, $\mathrm{CoP}$ allows us to examine the differences between groups (for example game development students and company representatives) and professions (for example programmers and graphic designers)

In 2018, Finnish game industry included a little over 200 game studios that employed altogether around 3,200 people. With the annual turnover of around 2,1 billion euros, Finland was placed within the top-three game developer countries in Europe by turnover [12].

Computer game development in Finland can be traced back to the very first computers in the country, but more serious game development efforts began to appear only when home computers gained popularity in the 1980s. For most people, game development remained largely a hobbyist endeavour, and many of the early companies had their connections to the computer subculture and the demoscene $[13,14,15]$. In this respect, the communal aspect has been crucial for the local industry from the beginning [16]. Also government funding has been used to support collaboration and information sharing between companies [17].

In the early days of the industry, communication between individual developers was fairly simple as the number of people making games was very modest and most people - at least in the capital region - knew each other. As the number of actors grew, need for more organized networking and social gatherings was identified. IGDA Finland Association, a local chapter of the International Game Developers Association, was founded in 2012 to support and bring together individual developers in a national level.

The International Game Developers Association, founded in the US in 1994, is a nonprofit organization with a mission to serve and support people who create games. These days, with a presence in almost 50 different countries, the association has spread its influence worldwide. The initial motivation behind the association was to provide individual game developers a channel for getting their voice through. While large publishers had their 
own associations, individual developers rarely had proper peer groups. While IGDA is committed to raising public awareness of game development as a profession and has its initiatives related to education and research, the central activity is to bring game developers together in large scale international conferences and smaller local events. [18]

The Finnish IGDA chapter works in close collaboration with two other associations, namely Suomen Pelinkehittäjät ry ("Finnish Game Developers Association") and local game development industry organization Neogames. Pelinkehittäjät association acts as the guardian of interests for the Finnish game development companies. Neogames also works to create synergies between different actors of the the videogame ecosystem be it game studios, other related companies or educational institutions. Direct cooperation between the organizations is visible in the distribution of information and joint events. While Pelinkehittäjät and Neogames are mostly interested in providing useful information and support for the companies, IGDA Finland can be seen to put more focus on individual game developers.

Recent research has highlighted how local game industry ecosystems can be seen as arenas for power struggles including simultaneously elements of competition and collaboration [19]. At the same time, prior research also suggests that the overall atmosphere in the IGDA Finland gatherings is relaxed and even well-known developers and CEOs are easily approachable [10]. According to this reading, the value of regular meetings is based on the way they succeed in bringing people together in a non-business manner. Prior studies, however, focus solely on meetings organized in Helsinki and leave the smaller hubs entirely unexamined. In addition to the activities in the capital area, IGDA Finland now has more than ten volunteer established local hubs in the country [20].

\section{Data and methodology}

The primary empirical data of the study consists of 12 thematic interviews with individuals who had visited a local IGDA gathering or other events hosted by the organization. Semi-structured interview is an apt venue for exploring how subjects experience and understand the lived world around them. It provides access to everyday activities of subjects, who in their own words describe their experiences and opinions [29]. Instead of anonymized data and blunt generalizations, we wanted to collect personal insights, tease out forms of situated knowledge central to particular communities, and to learn how the activities are contextualized in the everyday lives of different kinds of game makers. While the interviews were primarily focused on the local IGDA meetings, they also covered more general issues related to the local game development scenes. The questions covered the following key themes: overview of the local game development scene, IGDA meeting attendee history and motivations, social communication in meetings, learning related to meetings, overall benefits and shortcomings of the local IGDA activities.

The aim was to gather data from a diverse set of people, so the informants range from experienced game developers from both small and middle-sized game development companies to students with relatively little experience from the field. The professional roles of the informants include e.g. animator, CEO, graphical artist, operations manager, programmer and technical director. In the following subchapters, the respondents are separated by distinct IDs. Kajaani respondents have IDs starting with letter K and Tampere respondents are identified with the letter T. Before the actual study, three "beta" interviews were conducted to test the structure and the key themes. As already these interviews contain some valuable insights, we ended up including these first round responses in the dataset as well.

The full list of informants is as follows: 
1st round respondents:

- B1: Male, 32 years. Finnish. Student, part-time worker.

- B2: Male, 29 years. Finnish. Programmer, studio of ca. 10 employees

- B3: Male, 32 years. Finnish. Artist, part-time teacher.

Kajaani:

- K1: Male, 29 years. Finnish. CEO, studio of ca. 5 employees.

- K2: Male, 23 years. Finnish. 3rd year game development student

- K3: Male, 34 years. Finnish. Programmer, studio of ca. 10 employees.

- K4: Male, 56 years. Finnish. CEO, studio of over 50 employees

Tampere:

- T1: Male, 36 years. Finnish. Operations Manager, studio of over 20 employees.

- T2: Female, 29 years. Finnish. Graphical artist, unemployed, studying.

- T3: Male, 35 years. French. Technical Director, studio of over 20 employees.

- T4: Male, 35 years. German. Rigger/Animator, freelancer

- T5: Female, 22 years. Finnish. 2nd year game development student

The interviews were conducted both face-to-face and via phone and Skype-calls. The length of the interviews ranged from 25 to 60 minutes. Face-to-face interviews were recorded with a separate audio recorder with a smartphone serving as a backup recorder. Interviews done via phone and Skype calls were recorded with a call recorder application and the call recording functionality built in Microsoft Skype.

The recorded interviews were later transcribed for the analysis purposes. The analysis followed a qualitative approach in which the interviews were first carefully read and the contents were divided to loose thematic categories. Interviews were then coded according to the key themes and also the connections between themes were evaluated [30]. Direct quotes from the respondents were used to shed light into the individual experiences and to illustrate particularly interesting insights (English language excerpts were used as is. Finnish language quotes are translated by the authors). Concentrated summaries were created for both Kajaani and Tampere cases. These area-based summaries were then supplemented with detailed accounts brought up by the individual respondents.

The resulting comparisons between the two areas were done by evaluating the responses while keeping the geographical and historical differences in mind. Here we utilized the auto-ethnographic knowledge [31] one of the authors has collected as an IGDA volunteer. A total of five years of first-hand experience in various hub activities both in Kajaani and Tampere has significantly helped us in the selection of respondents and the design of interview questions. Being regularly present in the gatherings has also allowed us to observe the common practices of the attendees and how different groups of people interact with each other. This contextualized knowledge has helped us to provide needed background for the interview data, to problematize most obvious answers and to evaluate contradictory explanations. 


\section{Two local hubs: differences and similarities}

Together Tampere and Kajaani provide a nice overview of local hub activities outside the capital area. They were born around existing game development activities - including both game companies and game development related education programs. Hub meetings are normally organized in a monthly basis, although less frequently in the summer months when many people are in holidays. In this sense they are very similar to other smaller hubs in Finland. At the same time they also have their differences that are dicussed in the following chapter.

Located $600 \mathrm{~km}$ northeast from Helsinki, Kajaani (ca. 35,000 inhabitants) is the capital of Kainuu region (ca. 70,000 inhabitants). Kajaani University of Applied Sciences has been offering a large portfolio of game development studies for several years. As a result, Kajaani has one of the largest local student pools in the country that also affects the groups of people present in the local IGDA gatherings. In addition to Critical Force, the developer of Critical Ops that has over 50 employers in Kajaani, the lively start-up scene hosts over a dozen smaller game companies.

Tampere (ca. 230,000 inhabitants), in comparison, is the capital of Pirkanmaa region (ca. 500,000 inhabitants). Tampere city region is the second largest growth center in Finland and its business and cultural life benefits from the suitable location in the crossroads of the main traffic ways. Tampere has a long history of local game development and hosts the second largest game cluster in Finland with almost 30 companies employing over 200 game industry professionals [12]. In the following, we will analyze the local particularities more in detail.

\subsection{Kajaani hub}

In Kajaani, the IGDA hub was founded in 2014 and has been active ever since. An average gathering attracts a few dozen attendees, whereas a well-known visiting speaker can add to the number of participants. IGDA Kajaani Facebook group has over 120 members, although some of them are not based in the Kajaani area. Most of the attendees of the hub gatherings consist of local developers and the game development students from the Kajaani University of Applied Sciences. All interviewees in Kajaani classified themselves as regular attendees. General motivations for attending the gatherings were mostly uniform: wanting to see old friends and hearing about the news within the scene were the most common ones, with networking opportunities and meeting new people mentioned as well. Overall, the gatherings are seen as a good place to meet because of their open nature. This could indicate that even in a fairly small area as Kajaani, there is not that much interaction between the local game development companies and their employees. One of the interviewees, currently working in one of the local companies, had previous connections to the local game education institution and he was interested in hearing the stories of his previous students and how far they have gotten since graduation.

From the student point of view, the motivations for attending IGDA Finland Kajaani Hub gatherings has changed during the years. One interviewee stated that in the beginning of the studies, the main reason for attending the gathering was to network with people and to hear about the local companies and their work. As the studies progressed, the reason changed into being more about hearing news and building relationships with the companies.

All four interviewees agreed that having an active IGDA hub is beneficial to the local game development scene. The hub activities bring different groups of people together, provide a relaxed space for new connections to be made and make the game companies easily approachable. In the case of Kajaani, the IGDA hub has evolved from the previous, more spontaneous, game industry gatherings. One of the interviewees was responsible for the 
previous informal gatherings of the Kajaani game development scene. He sums the benefits of having a local hub as follows:

IGDA has changed things to the better, at least with the addition of speakers to the events. As the industry grows, it is natural for someone to take a full-time position in organising these events. Back then people were just spontaneously invited to join for a beer, it was easy since there were only 20 to 30 people. [K1]

The above quote shows how the growth of the local industry can sometimes make it more difficult to stay in touch with the other developers. Before the arrival of IGDA, local gatherings were mostly attended by the CEOs of the local game development companies as these people had been studying in the same programme. Also in the current IGDA events, attendees have a tendency of forming groups based on particular company or a study programme. This can lead to a situation in which the entire gathering consists of small, tight groups of people that can appear hard to get into. This seems to happen often with the new students from the local university:

New students often group up with other new students and do not talk to others unless encouraged to go and socialize with new people. Then again, it is up to the person if one wants to talk to strangers or not." [K2]

One of the mentioned benefits of the local hub is that it brings in people from outside Kajaani, further improving the possibilities for networking. For students, the hub activities are beneficial in many aspects. Firstly, the gatherings provide easily accessible grounds for making connections with any company representatives while making themselves known to the game development community. Secondly, active attendance in the events can strengthen one's professional identity and give an active impression to others working in the scene.

\subsection{Tampere hub}

The meetings of Tampere hub, founded in 2013, normally attract 100-150 attendees. IGDA Tampere Facebook group has over 900 members which nicely highlights that also people from other close-by cities frequently visit the hub meetings. The attendees to the IGDA Finland Tampere Hub always present a diverse set of reasons for visiting the gatherings. The main reason throughout the respondents turned out to be networking with other attendees. This was highlighted especially by those who were new to the area and looking to get into the local scene and by students looking for internship positions. A senior game developer explained his reasons for attending the gatherings after moving to the area:

They [motivations for attending] have changed as time passed. Originally when I moved here, I needed to find a team. I needed to make friends. I wanted to meet like-minded people who were interested in game development in the same way that I am. And through that, find a job. [T1]

In addition to networking, other common reasons were meeting old friends and hearing about their projects and current situation. While employees of a same studio meet each other regularly at work, they might not know what their friends and acquaintances in other companies are doing. For a newcomer, the crowd, often including industry veterans from well-known companies, can sometimes be a bit hard to enter.

Back when visiting the gatherings in Helsinki the main motivation was to get to know people from the industry and to understand what happens there. Then little by little the process has become more natural. At first, the visits were more about 'what is one supposed to do in these events', 'who to talk to' and 'in which table to sit'. [B1]

Learning something new about game development at an IGDA Tampere hub gathering was also commonly reported. The learning experiences were mostly related to receiving feedback on one's own work and seeing other people's projects. The so-called demo evenings were reported as the most effective forum for giving and 
receiving feedback. More experienced game developers happily gave constructive critique for student projects and the like.

\subsection{Comparisons between hubs}

The differences between Kajaani and Tampere are many, but so are the similarities. Both areas host an active game developer community and scene, supported by the local educational institutions. While Tampere has a longer history in game development with many successful companies over the years, Kajaani has also proven its worth as a location for game development.

For Kajaani, the very geographical location in Kainuu region in Eastern Finland poses some challenges. It is not always easy to attract speakers and sponsors to a hub clearly outside the main urban areas in the country.

Transportation takes time and accommodation needs resources, whereas in Tampere speakers and sponsors can be found within the area or easily invited e.g. from the capital area. The Kajaani hub enjoys the benefit of having a small, easily manageable community as well as the possibility of using the local University of Applied Sciences as leverage for getting guest speakers.

For the volunteers, the hubs are also different. The Kajaani hub's group of volunteers are mainly students from the University of Applied Sciences, who naturally have close ties with the university. This allows the IGDA Finland volunteers at the Kajaani hub to design the events from a student's perspective, resulting possibly in better learning experiences and helping students to connect with the local game development companies. The volunteers of the Tampere hub are mostly people from the local companies. Accordingly, the Tampere events and their program are designed more from the industry standpoint. Connections to the local educational institutions exist, but they are not actively utilized when looking for sponsors or speakers.

The layout of the venue used for the gatherings in both Kajaani and Tampere affects the atmosphere of the local IGDA gatherings. The current venue of the Kajaani hub gatherings, Rock House Kulma, is an open space with a sizable stage equipped with a projector and a screen. The stage is visible from almost every corner of the bar and the sound system can be easily utilised. Rock House Kulma is rather small for mass events, so space can become of an issue if there are over 100 attendees at a gathering.

In Tampere the common venue for the IGDA gatherings, Jack the Rooster, is more spacious, accommodating a larger crowd. The drawback of this space is that the main room of the bar does not feature a projector, nor a screen, so they must be brought in and set up by the Tampere hub volunteers. Jack the Rooster is also split into two different rooms instead of being a uniform space. The two separate rooms, of which the other is equipped with a separate karaoke booth, allows people not interested in the presentation to converse in peace without disturbing the presentation. The separate, usually less crowded room is also used as a demo-corner area during particular events in which companies and teams can represent their games and other activities to the visitors.

4.3.1 Attendee groups. The attendee demographics vary significantly in between the IGDA hubs of Kajaani and Tampere. Due to the large number of game development students present in the Kajaani University of Applied Sciences, the IGDA Finland Kajaani Hub gatherings are mostly populated by students specializing in game development and its different aspects. While there are many active game development companies in Kajaani, their presence in the gathering is rather small. These companies are utilized to gain speakers for the gatherings very much in the same way as in the Tampere hub. 
In the Tampere hub gatherings, the visitors are much more diverse, with most of the crowd comprising of representatives of companies of various sizes. The student attendance, however, has been on the rise in the Tampere hub gatherings. Tampere University of Applied Sciences students are well represented, whereas occasional students from Tampere University or e.g. Ahlman Vocational School also attend the meetings. More experienced students are reported to encourage younger students to attend the gatherings:

The school has not directly encouraged us to attend the gatherings, but older students have strongly recommended going to the gatherings. I myself as a tutor have tried to coerce my tutees to come to the gatherings, and at least three have attended. I have tried to spread the word about the usefulness of the gatherings. [T5]

This serves to show that students recognise the importance of attending the gatherings for the apparent benefits. Peer support for participating the events is important, as joining the experienced students can open up more chances for networking.

4.3.2. Motivations for attending the gatherings. The reasons for attending the local IGDA gatherings are unsurprisingly rather similar. Respondents both from Kajaani and Tampere state networking as their main reason for attending the events. This is understandable because of the nature of the industry. Most open positions in many companies are filled with candidates found via social networks. Networking and meeting people in the gatherings functions well for this purpose. Altogether four of informants had found their jobs and internship positions with the help of connections they have made in an IGDA gathering.

The job positions are not exactly handed out at the gatherings, however. Often the networking at a gathering is the first step in the progress of landing a job. Being present at the gathering and talking to people is a good way of making oneself known to potential employers. Showing one's work in the gatherings, especially at demo corner events, was seen as an effective way of getting noticed in the scene.

Giving and receiving feedback about one's own work appeared important for many respondents. The IGDA Finland Tampere hub seemed to be more active in organizing the so-called "demo evenings" or "demo corners" where companies of all sizes and student teams can showcase their products for others to evaluate. In Kajaani, these kinds of demo events were reportedly rare, and respondents from the area wished there to be more. Even without these kinds of events, many aspiring artists and developers were ready to present their work from their laptops or handheld devices to collect feedback and get some game testing experience.

The willfulness to help others is not uncommon in the game development industry. This is related to co-operative competition or "coopetition" [32] that is often seen typical of the Finnish video-game industry [10,11]. It is commonly seen that by supporting the people new to the industry, the local industry can grow stronger. This support will therefore help both parties and not result in one gaining an advantage over the other providing the information.

Other key reasons for attending hub events included hearing latest news, seeing old friends and giving or receiving feedback. Information flow within the industry is active, but not all company news is spread via their respective websites. Sponsored presentations are obviously one way to communicate the company's message to the visitors. Still, informal discussions were often found more important in learning about the latest projects. 


\section{Discussion: Hubs as communities of practice}

A recent industry-wide report suggests that "[t]he role of regional clusters and hubs is essential in the Finnish game industry ecosystem since they provide services, funding and an operational environment to start-ups and smaller companies in their respective regions" [12]. Our study puts the focus on the impact the local activities, and especially IGDA gatherings, can have on individual game makers. In this section, we will also return to the idea of community of practice and discuss the findings in the context.

Based on our data, individual motivation for entering the local meetings and different communities change over time. While networking seems to be the main motivation across the participants, more detailed motivations are often connected to the career phase. First the main purpose is to network and to learn to know people, but if the individual gets a job and advances in their career, the motivations change into more information sharing related aspects. This kind of "motivational evolution" was apparent in the interviews: developers already working in the industry do not often go to the events with job hunting in mind, but rather enter the gatherings to meet new people and to exchange information.

As our data shows, people in the gatherings often engage in small-talk about their trade and current projects, sharing tips and suggestions on how to tackle certain issues in any area of game development. The felt benefits are, however, seemingly different for people representing different roles in game development. Artists can readily showcase their work to their peers and potential employers, and programmers can provide insights to certain programming methods and sometimes even share small snippets of their code so that others can learn from their example. The same may not, however, apply to e.g. game designers who need at least a crude prototype to illustrate key mechanics or producers whose contribution is more difficult to define and demarcate.

In a first glance, emergence of communities of practice [26] comes close to how IGDA gatherings appear on the outside. The shared practices are numerous, centered around game development and game business. For Wenger, the communities are a platform for forming identities, learning and finding meaning. These activities "reify" the communal practice by "giving understanding a form". However, this reification does not primarily happen in the hub gatherings, as games themselves are not usually made during these events.

Learning within the community of practice - including e.g. learning the culture of the community, the tools of the trade, and the methods to use them in practice - is traditionally thought to happen during the time that one spends inside said community. While IGDA gatherings can include this aspect of learning, they rather initiate the process that then later continues outside the immediate meetings. The peer support aspect of the gatherings makes IGDA activities similar to "constellations of practice" also discussed by Wenger [26]. In these constellations, smaller communities of practice interact with each other despite their practices being very different from their peers.

It is not uncommon that while the participants may not necessarily share profound practices, they can still have shared interests. These communities of interest [21] can be more momentary but they can also be easier to access than long-lasting communities of practice that can sometimes be difficult for a newcomer to get into [33]. As discussed in the previous chapter, a new game development student looking to network with the local companies and experts can find it somewhat difficult to get started.

As is common in any events where there are a lot of people, attendees to an IGDA gathering often form small groups in which they mostly stay for the duration of the event. This group behaviour can be harmful especially to people new to the scene and to IGDA gatherings. In this respect, visiting speeches and presentations have an 
important role not only in passing the latest news but also in introducing at least a few people that can be easily approached.

In Wenger's (1998) original study, the formation of communities of practice took place inside a certain company. The communities initiated by IGDA events seem to exist between individual game development companies. The social bonds formed in the gatherings importantly extend learning and information sharing networks to people working in different companies.

\section{Conclusions}

Based on our findings, it seems clear that having a local hub and regular local events has an impact to the local game development scene. Promoting the flow of information within the local industry helps in many levels. For companies, these events provide a platform for recruitment and to showcase their work. For developers, hub meetings are about learning to know like-minded individuals and about strengthening the social networks locally and regionally. IGDA hubs also promote the local game development networks to people outside the industry, allowing people to learn more about game making.

If the representatives of known local company do not actively and visibly attend the events, this can raise some suspicions. As one of the respondents stated: "Sometimes there are companies that do not usually show up in IGDA. It makes you think that does such a company even exist." [B3]. In this respect, being present in an IGDA gathering is directly connected to the public image of a company. Dedicating time to attend a gathering can therefore be an easy way of gaining publicity and credibility on the local level. Simple means as wearing the company hoodie or shirt (actively utilized e.g. by Colossal Order and Random Potion employees at the Tampere hub gatherings) not only provides immediate exposure to the company but can also contribute to the individual level identity work.

The felt benefits for individuals seem to vary at least based on the career phase and the expert role. Overall, aggregating different insights and accelerating the flow of information seems important for people in very different positions. While demo-corner events, sponsored talks or associated seminars provide important learning opportunities, many informants still underline the importance of just hanging out with others who are willing to give advice and share information about their trade.

We hope that this study can provide some preliminary basis for studying local game development scenes and supporting initiatives like local IGDA hubs. Similar studies from other countries would give better understanding of regional differences and more ground for comparisons. It would also be interesting to compare the local information sharing practices with other creative industries.

It is also clear that there were some issues where the informants easily followed to dominant narrative of the Finnish digital game industry as a big happy family and maybe did not want to break the common consensus. Silenced topics could include for example rivalries between companies, recruitments to the competing companies, or tensions in equality issues. These are issues that need to be addressed more in detail in the future.

Finally, we are aware that only people who actively participate in IGDA events were interviewed for this study. This kind of "survivor-bias" is common for game industry studies and in the future we would like to see studies also involving people who decide not to attend local meetings or have entirely moved away from game 
development. Forthcoming studies should also look at active game development areas who do not host an IGDA hub, and how they compare to the ones in which a local hub has been established.

\section{ACKNOWLEDGMENTS}

We want to express our gratitude to professor J.Tuomas Harviainen for his invaluable input in the early phases of this project. Olli Sotamaa's work was supported by the Academy of Finland project Center of Excellence in Game Culture Studies (CoE-GameCult, 312395).

\section{REFERENCES}

[1] B. Keogh. 2019. From aggressively formalised to intensely in/formalised: accounting for a wider range of videogame development practices. Creative Industries Journal, 12(1), 14-33.

[2] A. Kerr and A. Cawley. 2012. The spatialisation of the digital games industry: Lessons from Ireland. International Journal of Cultural Policy, 18(4), 398-418.

[3] U. Sandqvist. 2012. The development of the Swedish game industry: a true success story? In P. Zackariasson and T. Wilson (Eds.), The video game industry: formation, present state, and future. Routledge, New York, 134-156.

[4] J. Banks and S. Cunningham. 2016. Creative destruction in the Australian videogames industry. Media International Australia, 160(1), 127-139.

[5] J. Švelch. 2018. Gaming the iron curtain: How teenagers and amateurs in communist Czechoslovakia claimed the medium of computer games. MIT Press, Cambridge (Mass.).

[6] F. Parker and J. Jenson. 2017. Canadian indie games between the global and the local. Canadian Journal of Communication, 42(5), 867-891.

[7] A.M. Ozimek. 2019. Outsourcing digital game production: The case of Polish testers. Television \& New Media, 20(8), 824-835.

[8] C. O'Donnell. 2014. Developer's dilemma: The secret world of videogame creators. MIT Press, Cambridge (Mass.).

[9] J.R. Whitson. 2018. What Can We Learn From Studio Studies Ethnographies? A "Messy" Account of Game Development Materiality, Learning, and Expertise. Games and Culture.

[10] T.-M. Karjalainen, M.J. Lehtonen and J. Niipola. 2014. Pelisilmää, Sävelkorvaa: Tarinoita suomalaisesta peli- ja musiikkiviennistä. Talentum Media Oy, Helsinki.

[11] E. Lappalainen. 2016. The realm of games: How a small Nordic nation became an industry giant. Neogames Finland Association, Helsinki.

[12] Neogames, 2019. The game industry of Finland: Report 2018. http://www.neogames.fi/wpcontent/uploads/2019/04/FGIR-2018-Report.pdf

[13] P. Saarikoski and J. Suominen. 2009. Computer Hobbyists and the Gaming Industry in Finland. IEEE Annals of the History of Computing, 30(3), 20-33.

[14] H. Tyni and O. Sotamaa. 2014. Assembling a Game Development Scene? Uncovering Finland's Largest Demo Party. GAME 3/2014: 109-19.

[15] K. Jørgensen, U. Sandqvist, and O. Sotamaa. 2017. From hobbyists to entrepreneurs: On the formation of the Nordic game industry. Convergence 23(5), 457-76.

[16] O. Sotamaa, H. Tyni, S. Toivonen, T. Malinen, and E. Rautio. 2011. New Paradigms for Digital Games: The Finnish Perspective. Future Play Project, Final Report. TRIM Research Reports. University of Tampere, Tampere.

[17] O. Sotamaa, K. Jørgensen and U. Sandqvist. 2019. Public game funding in the Nordic region. International Journal of Cultural Policy.

[18] IGDA. 2019. History of the IGDA. https://www.igda.org/page/history 
[19] M.J. Lehtonen, A. Ainamo and J.T. Harviainen. 2019. The four faces of creative industries: visualising the game industry ecosystem in Helsinki and Tokyo. Industry and Innovation.

[20] IGDA Finland. 2019. https://www.igda.fi/hubs

[21] U. Bußmann. 2014. Organisational cultures: Networks, clusters, alliances. Diplomica Verlag, Hamburg.

[22] H. Izushi and Y. Aoyama. 2006. Industry Evolution and Cross-Sectoral Skill Transfers: A Comparative Analysis of The Video Game Industry in Japan, the United States, and the United Kingdom. Environment and planning A, 38(10), 1843-1861.

[23] M. Toftedahl, B. Marklund, H. Engström and P. Backlund. 2016. Global influences on regional industries: Game development in Nordic countries, China, and India. Proceedings of Chinese DiGRA 2016.

[24] D. Grandadam, P. Cohendet and L. Simon. 2013. Places, spaces and the dynamics of creativity: The video game industry in Montreal. Regional Studies 47(10), 1701-14.

[25] J. Lave and E. Wenger. 1991. Situated Learning: Legitimate Peripheral Participation. Cambridge University Press, Cambridge.

[26] E. Wenger. 1998. Communities of practice: Learning, meaning, and identity. Cambridge: Cambridge University Press.

[27] A. Bourhis, L. Dubé and R. Jacob. 2005. The Success of Virtual Communities of Practice: The Leadership Factor. The Electronic Journal of Knowledge Management, 3(1), 23-34.

[28] J. Roberts. 2014. Community and the dynamics of spatially distributed knowledge production. In R. Rutten, P. Benneworth, D. Irawati and F. Boekema (Eds.), The social dynamics of innovation networks. Routledge, London, 179-200.

[29] S. Kvale. 2007. Doing Interviews. Sage, London \& Thousand Oaks.

[30] U. Kuckartz. 2014. Qualitative Text Analysis: A Guide to Methods, Practice \& Using Software. Sage, London \& Thousand Oaks.

[31] Van Maanen, John. 2011. Tales of the field: On writing ethnography. Second edition. University of Chicago Press, Chicago \& London.

[32] M. Bengtsson and S. Kock. 2000. "Coopetition" in business networks to cooperate and compete simultaneously. Industrial Marketing Management 29(5): 411-426.

[33] M. Ackerman, V. Pipek \& V. Wulf (Eds.). 2003. Sharing Expertise: Beyond Knowledge Management. MIT Press, Cambridge (Mass.). 